\title{
Validation of simple dichotomous self-report on prenatal alcohol and other drug use in women attending midwife obstetric units in the Cape Metropole, South Africa
}

Clinical Ethics 2020, Vol. 15(4) I8I-186 (C) The Author(s) 2020 Article reuse guidelines: sagepub.com/journals-permissions DOI: 10.1 177//477750920928885 journals.sagepub.com/home/cet (S)AGE

\author{
Petal Petersen Williams ${ }^{1,2}\left(\mathbb{D}\right.$, Catherine Mathews ${ }^{3,4}$, \\ Esmé Jordaan $^{5,6}$ D, Yukiko Washio ${ }^{7}$, Mishka Terplan ${ }^{8}$ and \\ Charles DH Parry ${ }^{1,9}$
}

\begin{abstract}
Background: This paper examines the degree of agreement among simple dichotomous self-report, validated screening results, and biochemical screening results of prenatal alcohol and other drug use among pregnant women.

Method: Secondary analysis was conducted on a cohort of pregnant women 16 years or older, presenting for prenatal care in the greater Cape Town, South Africa. Dichotomous verbal screening is a standard of care, and pregnant patients reporting alcohol and other drug use in dichotomous verbal screenings were asked to engage in screening using the Alcohol Smoking and Substance Involvement Screening Test (ASSIST) and urinalysis.

Results: Significant agreements between dichotomous and ASSIST scores were observed $(K=0.73-0.76)$. A higher rate of self-reported $(36.9 \%)$ alcohol use was detected, relative to urine screening $(19.6 \%)$ with a predictive value of 34.9 ; while underreporting of illicit substance use was observed (3.6\% self-report vs. $8.8 \%$ urine screening) with an overall predictive value of 50.0 .

Conclusion: Dichotomous verbal screening was considered valid after comparison with the ASSIST; however, combined use with urine screenings can be recommended especially for identifying illicit substance use in order to accurately detect alcohol and other drug use in pregnancy, so that women can be identified and referred for appropriate interventions where needed.
\end{abstract}

\section{Keywords}

Validity, ASSIST, pregnancy, alcohol and other drug use, South Africa

\section{Introduction}

Prenatal substance use (illicit drugs, alcohol, and tobacco) is one of the largest modifiable risk factors for significant gestational complications for both women and their newborns. ${ }^{1-4}$ Polysubstance use

\footnotetext{
'Alcohol, Tobacco \& Other Drug Research Unit, South African Medical Research Council, Tygerberg, South Africa

${ }^{2}$ Department of Psychiatry and Mental Health, University of Cape Town, Cape Town, South Africa

${ }^{3}$ Health Systems Research Unit, South African Medical Research Council, Tygerberg, South Africa

"Women's Health Research Unit, School of Public Health and Family Medicine, University of Cape Town, Cape Town, South Africa

${ }^{5}$ Biostatistics Unit, South African Medical Research Council, Tygerberg, South Africa
}

further increases the risk for adverse pregnancy outcomes including miscarriage, low birth weight, preterm

\footnotetext{
${ }^{6}$ Statistics and Population Studies Department, University of the Western Cape, Bellville, South Africa

${ }^{7}$ Substance Use, Gender and Applied Research, Research Triangle Park, NC, USA

${ }^{8}$ Department of Obstetrics and Gynecology, Division of General Obstetrics and Gynecology, Virginia Commonwealth University, VA, USA ${ }^{9}$ Department of Psychiatry, Stellenbosch University, Tygerberg, South Africa
}

\section{Corresponding author:}

Petal Petersen Williams, Alcohol, Tobacco \& Other Drug Research Unit, Medical Research Council, Francie van Zijl Drive, Tygerberg 7505, South Africa.

Email: petal.petersen@mrc.ac.za 
birth, stillbirth, and neurocognitive disorders. ${ }^{1-5}$ Maternal smoking, alcohol use, and drug use, each increased the likelihood of having neonatal morbidity including impaired Apgar scores ( $<7$ at 5 -min postpartum), neonatal resuscitation, and neonatal intensive care unit admission. ${ }^{6}$ While studies among certain populations of pregnant women attending antenatal services in the Western Cape have reported on rates of substance use, ${ }^{7-9}$ to date, national prevalence of prenatal alcohol and other drug (AOD) use is not known in South Africa, unlike in the U.S. ${ }^{10}$ However, locally, the prevalence data of AOD use exist for overall populations. ${ }^{11,12}$

Discrepancies between self-reported rate of AOD use and biochemically verified use have been well documented, in which self-reported AOD use generally demonstrated low sensitivity relative to biomarkers, ${ }^{13-15}$ in part due to fear of judgment. ${ }^{16}$ Nevertheless, biochemical screenings do not necessarily demonstrate clinical efficacy nor may they be ethically acceptable due to the legal implications of prenatal substance use, at least in the U. S. ${ }^{17}$ Although a severely punitive approach such as loss of child custody does not happen in South Africa, ${ }^{18}$ fear of exposure to stigmatization by healthcare providers may increase under-reported AOD use among pregnant women when asked in healthcare settings. ${ }^{19}$

A combined use of biochemical screenings with a standard self-report instrument (such as the Alcohol Smoking and Substance Involvement Screening Test; ASSIST) is encouraged for identifying substance use in pregnant women: ${ }^{17}$ however, little international empirical evidence supports this notion so far. For example, a previous report of a cross-sectional survey at a community-based prenatal clinic in South Africa showed that biochemical screening (i.e. urinalysis) detected a higher prevalence of prenatal drug use $(8.8 \%)$ compared to the self-reported dichotomous prevalence $(3.6 \%)$, while the opposite trend was found with prenatal alcohol use (i.e. $19.6 \%$ vs. $36.9 \%) .{ }^{8}$ Recent evidence with a large U.S. cohort of pregnant women also showed that simple, dichotomous self-report on prenatal substance, alcohol, and tobacco use can still be useful to predict adverse birth outcomes and start the conversation with obstetric providers on substance use during pregnancy. ${ }^{20}$

The current study quantitatively explored the degree of agreement among simple dichotomous self-report, validated screening results, and biochemical screening results in a South African cohort attending prenatal care, in order to determine the level of validity with the simple dichotomous self-report on prenatal AOD use.

\section{Methods}

The secondary analysis was based on a cohort of consenting pregnant women 16 years or older, presenting to community-based clinics called midwife obstetric units (MOUs) in the greater Cape Town, a city in the Western Cape region of South Africa. The procedure of collecting demographic status and prenatal substance and alcohol use has been stated elsewhere; ${ }^{8}$ however, briefly, each participant received reimbursement for their time and participation in the form of a chain store voucher to the value of R50 (approximately \$6). They were also provided with a resource list. Ethical approval to conduct the research was obtained from the Faculty of Health Sciences Research Ethics Committee at the University of Cape Town. Permission to conduct the survey in the MOUs was obtained from the Western Cape Department of Health.

\section{Measures of substance use}

Three means of assessing prenatal AOD use were compared to determine the validity of simple dichotomous self-report on prenatal substance use.

(1) Dichotomous self-report where women were asked about alcohol or drug use during their current pregnancy or in the three months before they knew they were pregnant. These questions resemble current standard care during intake, where pregnant women are asked direct questions about current tobacco, alcohol, and drug use, (2) a validated assessment based on the Alcohol, Smoking and Substance Involvement Screening Test (ASSIST), and (3) urine screening. The ASSIST is a brief screening questionnaire consisting of eight items and was developed by the WHO and an international team of substance use researchers as a simple method of screening for hazardous, harmful and dependent use of alcohol, tobacco, and other psychoactive substances. The ASSIST was designed to screen for substance abuse in primary care settings, and pregnant women have been recommended as a target group suitable for an ASSIST screening program by the developers of this tool. ${ }^{21}$ A validity study conducted with 1047 subjects from seven countries, including Australia, Brazil, India, Thailand, the UK, USA, and Zimbabwe, showed that the ASSIST had good concurrent, construct, predictive, and discriminative validity. ${ }^{22}$

\section{Data analysis}

Appropriate sampling weights were determined according to the study design to generalize the results to the described population of pregnant women. A survey analysis was conducted, using appropriate weights for the proportional allocation of women, to estimate the reported drug and alcohol prevalence and 95\% confidence limits applied to the sample. A finite population correction was also used. 
First, agreement between the self-reported substance use using simple, dichotomous questions (yes/no), and the substance use measured indirectly using the ASSIST relating to the last three month's substance use (aggregated responses; yes = once or twice, monthly, weekly, daily; no $=$ never or not in the past three months) was measured by the kappa statistic and $95 \%$ confidence interval. The kappa values were calculated on the weighted values in the $2 \times 2$ tables. To indicate the strength of the agreement, the standards of Landis and Koch were used: $<0.00=$ poor; $0.00-0.20=$ slight; $0.21-0.40=$ fair; $0.41-0.60=$ moderate; $\quad 0.61-0.80=$ substantial; $\quad$ and $0.81-1.00=$ almost $_{\text {perfect. }}{ }^{23}$

Second, agreement between the responses to the ASSIST and urinalysis results was examined, looking at the sensitivity, specificity, positive predicted value (PPV), and the negative predicted value (NPV) with $95 \%$ confidence intervals were reported as measures of validity. The analyses were undertaken for cannabis, heroin, methamphetamine, and benzodiazepine as well as for all drugs combined. For the urinalysis, the standardized cut-off values for the individual drugs were used to indicate positive use of the specific drug. The observed frequency tables were reported and thereafter the $2 \times 2$ tables. The measures of validity were calculated for the weighted values in the $2 \times 2$ tables. Analyses were conducted using SAS version 9.4.

\section{Results}

\section{Characteristics of the sample}

In total, 5231 pregnant women screened for AOD use during their current pregnancy and/or in the three months before they knew they were pregnant. Of these, $684(13.1 \%)$ were intentionally sub-sampled and completed ASSIST screening and provided a urine sample for biological screening. Self-reported alcohol and drug use were compared with urine screens. The 684 participants had a mean age of 26 years $(\mathrm{SE}=0.25) ; 60.3 \%$ were Black African, $39.1 \%$ coloured (of mixed-race ancestry), and $1.6 \%$ White or Asian. Ninety-two percent had some secondary education, $66.9 \%$ were never married/single, and $56.9 \%$ unemployed. The majority of participants had five or more of the listed assets in their homes $(75.9 \%)$ indicating higher socio-economic status compared to those with four or less items. Approximately twothirds were multiparous. Over half the participants reported that their current pregnancy was unplanned $(68.2 \%)$, and the mean gestational age at the booking visit was 18.9 weeks with the majority of women booking in their second trimester (57.4\%) (Table 1).
Table I. Participant demographic characteristics $(n=684)$.

\begin{tabular}{|c|c|c|}
\hline & $\%$ & SE \\
\hline \multicolumn{3}{|l|}{ Age } \\
\hline $16-19$ & 14.7 & 1.48 \\
\hline $20-29$ & 62.9 & 2.08 \\
\hline $30-39$ & 19.5 & $\mathrm{I} .73$ \\
\hline $40-49$ & 2.8 & 0.73 \\
\hline \multicolumn{3}{|l|}{ Race } \\
\hline Black African & 60.3 & 1.70 \\
\hline Coloured & 39.1 & 1.71 \\
\hline White/Asian & 1.6 & 0.55 \\
\hline \multicolumn{3}{|l|}{ Education } \\
\hline Primary & 3.5 & 0.79 \\
\hline Secondary & 92.0 & 1.16 \\
\hline Tertiary-incomplete & 1.9 & 0.62 \\
\hline Tertiary-complete & 2.6 & 0.65 \\
\hline \multicolumn{3}{|l|}{ Marital status } \\
\hline Legally married & 18.9 & 1.65 \\
\hline Traditionally married & 7.7 & 1.15 \\
\hline Living together & 4.1 & 0.84 \\
\hline Never married/single & 66.9 & 2.00 \\
\hline Divorced/separated/widowed & 2.4 & 0.68 \\
\hline \multicolumn{3}{|l|}{ Employment status } \\
\hline Unemployed & 56.9 & 2.14 \\
\hline Employed full-time & 23.7 & 1.86 \\
\hline Employed part-time/self-employed & 12.0 & 1.43 \\
\hline Student & 7.4 & 1.10 \\
\hline \multicolumn{3}{|l|}{ Socio-economic status } \\
\hline Low (four or less items) & 24.1 & $\mathrm{I} .78$ \\
\hline High (five or more items) & 75.9 & 1.78 \\
\hline \multicolumn{3}{|l|}{ Parity } \\
\hline 0 & 34.0 & 2.00 \\
\hline I & 35.1 & 2.10 \\
\hline 2 & 19.6 & 1.70 \\
\hline 3 & 8.2 & 1.20 \\
\hline$\geq 4$ & 3.1 & 0.70 \\
\hline \multicolumn{3}{|l|}{ Planned pregnancy } \\
\hline Yes & 31.8 & 2.10 \\
\hline No & 68.2 & 2.10 \\
\hline \multicolumn{3}{|l|}{ Gestational age } \\
\hline 0-12 weeks (first trimester) & 22.8 & 1.90 \\
\hline 13-24 weeks (second trimester) & 57.4 & 2.30 \\
\hline $25-40$ weeks (third trimester) & 19.8 & 1.80 \\
\hline
\end{tabular}

\section{Prevalence of $A O D$ use}

An estimation of the prevalence of substance use was conducted, taking into account stratified proportional allocation, adjusting variance for stratified proportional sampling. Three assessments of drug use were used to estimate prevalence: dichotomous self-report in the screened sample $(N=5231)$; an assessment based on the Alcohol, Smoking and Substance Involvement Screening Test (ASSIST) $(N=684)$; and biological urine tests $(N=592)$. Ninety-five urine samples were not tested for any $(n=34)$ or some $(n=61)$ substances 
because of a fault of the testing laboratory, which accounted for $12.2 \%$ of the total samples.

Dichotomous self-report $(N=5231)$ showed that $3.6 \%(95 \%$ CI: 3.1-4.0) reported at least one illicit substance use and 36.9\% (95\% CI: 35.6-38.1) reported alcohol use. ASSIST screening $(N=684)$ showed that $4.7 \%$ reported at least one illicit substance use and $36.8 \%$ reported alcohol use. Urinalyses $(N=592)$ showed that $8.8 \%(95 \% \mathrm{CI}: 6.7-10.9)$ tested positive for at least one illicit substance and 19.6\% (95\% CI: 16.3-22.8) tested positive for alcohol use.

\section{Agreement between dichotomous and ASSIST screenings}

The overall measure of agreement between the dichotomous self-reported AOD rate and AOD rate screened by ASSIST was statistically significant $(\mathrm{K}=0.76 ; 95 \%$ CI $0.71-0.81$ and $\mathrm{K}=0.73$; $95 \%$ CI $0.59-0.86$ for alcohol and drug use, respectively), indicating a strong agreement between the two means of verbal screenings.

\section{Agreement between dichotomous and urinalyses}

Urinalyses showed the rate of illicit substance use that was higher than dichotomous self-report $(3.6 \%$ vs. $8.8 \%$ ), and the rate of alcohol use that was lower than dichotomous self-report ( $36.9 \%$ vs. $19.6 \%$ ).

\section{Agreement between ASSIST and urine screenings}

Table 2 shows agreements between ASSIST and urine screening with respect to sensitivity, specificity, and predictability. The weighted sensitivity (the proportion of self-reports of alcohol use among those testing positive for alcohol use) of self-report for alcohol use using the ASSIST as compared to biomarkers was $58.5 \%$ (CI: 48.9-68.1), and the weighted specificity (the proportion of self-reports of no alcohol use among those testing negative for alcohol use) was $68.7 \%$ (CI: 63.973.6). The positive predictive value, which indicates how likely someone with a positive test result is to actually have the characteristic or be a user of alcohol, was $34.9 \%$.
The weighted sensitivity of self-report for other drug use was $22.5 \%$, and the specificity was $97.5 \%$. The PPV was $50.0 \%$. The weighted sensitivity for self-report of Cannabis use compared to biomarkers was $51.4 \%$ (95\% CI: $27.8-74.9$ ), and the specificity was $98.4 \%$ (95\% CI: 97.7-99.2) with the PPV of $37.6 \%$. Sensitivity analysis was not conducted for heroin use due to the small sample size $(n=5)$ with specificity of $99.9 \%$. The weighted sensitivity of self-report of methamphetamine was $13.3 \%$, and the specificity was $98.5 \%$ with the PPV of $46.8 \%$. No participant reported benzodiazepine use (Table 2).

\section{Discussion}

The current study showed that simple, dichotomous self-report showed a good agreement with ASSIST verbal screening. This indicates that simple, dichotomous self-report is a reliable means of detecting prenatal AOD use among pregnant women in the Western Cape region of South Africa. However, ASSIST identified more alcohol use and less illicit drug use compared to urine screenings. This indicates that pregnant women in the Western Cape region of South Africa are willing to disclose alcohol use, but tend to underreport drug use.

Data from studies conducted in the USA show that pregnant women are more likely to underreport alcohol use during pregnancy for the concern of stigmatization. ${ }^{16}$ Although public health messaging regarding harmful effects of drinking during pregnancy is available, and a brief intervention to educate pregnant mothers has been effective with a South African cohort, ${ }^{24}$ socioeconomically disadvantaged pregnant women enter late in prenatal care as found in this study. The reasons for this are complex and vary but includes importantly women's perceptions of quality of care, which influences their health seeking practices. ${ }^{25}$ It should be noted that underreporting of prenatal alcohol use might increase as the norm around perceived harm of prenatal alcohol use changes.

Contrary to reporting alcohol use, report of illicit substance use or noncompliance with a treatment plan

Table 2. Sensitivity, specificity, positive predictive value, and negative predictive value between ASSIST and urine screenings for alcohol and each substance.

\begin{tabular}{|c|c|c|c|c|c|c|c|c|}
\hline Substance & $\begin{array}{l}\text { Weighted } \\
\text { sensitivity }\end{array}$ & $95 \% \mathrm{Cl}$ & $\begin{array}{l}\text { Weighted } \\
\text { specificity }\end{array}$ & $95 \% \mathrm{Cl}$ & PPV & $95 \% \mathrm{Cl}$ & NPV & $95 \% \mathrm{Cl}$ \\
\hline Alcohol & 58.5 & $48.9-68.1$ & 68.7 & 63.9-73.6 & 34.9 & $27.5-42.2$ & 85.3 & $81.5-89.0$ \\
\hline Cannabis & 51.4 & $27.8-74.9$ & 98.4 & $97.7-99.2$ & 37.6 & $21.0-54.1$ & 99.1 & $98.4-99.8$ \\
\hline Heroin & NA & - & 99.9 & $99.9-100.0$ & - & - & - & - \\
\hline Methamphetamine & 13.3 & $8.5-18.1$ & 98.5 & $97.5-99.5$ & 46.8 & $29.9-63.8$ & 91.8 & $89.3-94.2$ \\
\hline Benzodiazepine & NA & - & 99.9 & $99.7-99.9$ & - & - & - & - \\
\hline Drugs overall & 22.5 & $15.2-29.9$ & 97.5 & $96.3-98.7$ & 50.0 & $36.0-64.0$ & 91.9 & $89.4-94.3$ \\
\hline
\end{tabular}


among mothers can result in rather severe legal consequences such as loss of child custody and incarceration in the U.S. ${ }^{17}$ This is not the case with South Africa to date, ${ }^{18}$ and potential consequence of reported illicit drug use is assignment of case management by a social worker; however, stigmatization by healthcare professionals on prenatal illicit drug use ${ }^{19}$ is potentially the reason of underreported drug use in the current study. Due to low disclosure rate of drug use among pregnant women, the combined verbal and urine screenings are recommended to determine the national prevalence of prenatal drug use; while, verbal screening only captures the prevalence of prenatal alcohol use. However, questions around the affordability of this remain and the availability of resources to respond appropriately.

A few limitations are noted in the current study. Not all pregnant patients volunteered for the study; therefore, this was considered a convenient sample. Not all participants who were screened with ASSIST had their urines tested due to laboratory issues with some samples being inadvertently destroyed.

The current study contributes to the existing literature in the following ways: (1) ASSIST verbal screening demonstrated a high agreement with dichotomous verbal screening with a South African cohort which implies that we can continue using simple questioning about use (yes/no); (2) the rate of self-reported prenatal alcohol use was higher than that of urine screening with the cohort, contrary to the U.S.; (3) the rate of self-reported illicit drug use was lower than that of urine screening with the cohort, consistent with the literature in the U.S.

In summary, the current study examined agreements between dichotomous verbal screening, validated verbal screening, and urine screening in prenatal AOD use with a South African cohort. High rate of self-reported prenatal alcohol use was observed; however, illicit drug use was underreported. Dichotomous verbal screening was considered valid after comparison with the validated verbal screening method; however, combined use with urine screenings can be recommended especially for identifying underreported substances in order to accurately detect AOD use in pregnancy, so that women can be identified and referred for appropriate interventions where needed.

\section{Authors' Note}

Mishka Terplan is now affiliated with Friends Research Institute, Baltimore, MD, USA.

\section{Authors' contributions}

PPW designed the study and wrote the protocol. CP and CM made considerable contributions to the design of the study and assisted with the protocol development. EJ and PPW undertook the analysis. YW and MT provided expertise and guidance in addressing issues around validity in mapping out a manuscript. PPW and YW wrote the first draft of the manuscript. All authors contributed to and have approved the final manuscript.

\section{Acknowledgements}

The contents are solely the responsibility of the authors and do not necessarily represent the official views of the CDC or PEPFAR. The CDC had no further role in the study design; in the collection, analysis and interpretation of data; in the writing of the report; or in the decision to submit the paper for publication.

\section{Declaration of conflicting interests}

The authors declared no potential conflicts of interest with respect to the research, authorship, and/or publication of this article.

\section{Funding}

The authors disclosed receipt of the following financial support for the research, authorship, and/or publication of this article: The research was funded by the US President's Emergency Fund for AIDS Relief (PEPFAR) through the US Centers of Disease Control and Prevention (CDC) under the terms of 5U2GPS001137-05. The research was also supported by the South African Medical Research Council.

\section{ORCID iDs}

Petal Petersen Williams (D) https://orcid.org/0000-0001-55352458

Esmé Jordaan (D) https://orcid.org/0000-0002-0361-3473

\section{References}

1. Aliyu MH, Wilson RE, Zoorob R, et al. Prenatal alcohol consumption and fetal growth restriction: potentiation effect by concomitant smoking. Nicotine Tob Res 2009; 11: 36-43.

2. Bailey BA, McCook JG, Hodge A, et al. Infant birth outcomes among substance using women: why quitting smoking during pregnancy is just as important as quitting illicit drug use. Matern Child Health J 2012; 16: 414-422.

3. CDC. Alcohol use in pregnancy, http://m.cdc.gov/en/ HealthSafetyTopics/LifeStagesPopulations/Pregnancy/ alcoholUse (2013, accessed 19 June 2017).

4. Mark K, Desai A and Terplan M. Marijuana use and pregnancy: prevalence, associated characteristics, and birth outcomes. Arch Womens Ment Health 2016; 19: 105-111.

5. Quesada O, Gotman N, Howell HB, et al. Prenatal hazardous substance use and adverse birth outcomes. J Matern Fetal Neonatal Med 2012; 25: 1222-1227.

6. Burstyn I, Kapur N and Cherry NM. Substance use of pregnant women and early neonatal morbidity: where to focus intervention? Can J Public Health 2010; 101: 149-153.

7. Onah MN, Field S, van Heyningen T, et al. Predictors of alcohol and other drug use among pregnant women in a 
peri-urban South African setting. Int J Ment Health Syst 2016; 10: 38.

8. Petersen Williams $\mathrm{P}$, Jordaan E, Mathews C, et al. Alcohol and other drug use during pregnancy among women attending midwife obstetric units in the Cape Metropole, South Africa. Adv Prev Med. Epub ahead of print 3 February 2014. DOI: 10.1155/2014/871427.

9. Vythilingum B, Roos A, Faure SC, et al. Risk factors for substance use in pregnant women in South Africa. $S \mathrm{Afr}$ Med J 2012; 102: 851-854.

10. Samhsa C. Results from the 2015 National Survey on Drug Use and Health: detailed tables, www.samhsa.gov/ data/sites/default/files/NSDUH-DetTabs-2015/NSDUH-

DetTabs-2015/NSDUH-DetTabs-2015.htm (2015, accessed 22 May 2017).

11. Peltzer K, Davids A and Njuho P. Alcohol use and problem drinking in South Africa: findings from a national population-based survey. Afr J Psychiatr 2011; 14: 30-37.

12. Peltzer K, Ramlagan S, Johnson BD, et al. Illicit drug use and treatment in South Africa: a review. Subst Use Misuse 2010; 45: 2221-2243.

13. Bessa MA, Mitsuhiro SS, Chalem E, et al. Underreporting of use of cocaine and marijuana during the third trimester of gestation among pregnant adolescents. Addict Behav 2010; 35: 266-269.

14. El Marroun $\mathrm{H}$, Tiemeier $\mathrm{H}$, Jaddoe VWV, et al. Agreement between maternal cannabis use during pregnancy according to self-report and urinalysis in a population-based cohort: the Generation R study. Eur Addict Res 2011; 17: 37-43.

15. Garg M, Garrison L, Leeman L, et al. Validity of selfreported drug use information among pregnant women. Matern Child Health J 2016; 20: 41-47.

16. Muggli E, Cook B, O'Leary C, et al. Increasing accurate self-report in surveys of pregnancy alcohol use. Midwifery 2015; 31: e23-e28.

17. Terplan $\mathbf{M}$ and Minkoff $H$. Neonatal abstinence syndrome and ethical approaches to the identification of pregnant women who use drugs. Obstet Gynecol 2017; 129: $164-167$.

18. Gardner J. Should drinking during pregnancy be criminalised to prevent fetal alcohol spectrum disorder? $S$ Afr $J$ Bioeth Law 2016; 9: 26.

19. Petersen Williams P, Petersen Z, Sorsdahl K, et al. Screening and brief interventions for alcohol and other drug use among pregnant women attending midwife obstetric units in Cape Town, South Africa: a qualitative study of the views of health care professionals. $J$ Midwifery Womens Health 2015; 60: 401-409.

20. Washio Y, Goldstein ND, Butler R, et al. Self-report measure as a useful tool to identify prenatal substance use and predict adverse birth outcomes. Clin Ethics 2018; 13: 137-142.

21. Henry-Edwards S, Humeniuk R, Ali R, et al. The Alcohol, Smoking and Substance Involvement Screening Test (ASSIST): Guidelines for Use in Primary Care. Geneva: World Health Organization: Geneva.

22. Humeniuk R and Ali R. Validation of the Alcohol, Smoking, and Substance Involvement Screening Test (ASSIST) and Pilot Brief Intervention: A Technical Report of Phase II Findings of the WHO ASSIST Project. Geneva: World Health Organization (WHO), 2006.

23. Landis JR and Koch GG. The measurement of observer agreement for categorical data. Biometrics 1977; 33: 159-174.

24. Rendall-Mkosi K, Morojele N, London L, et al. A randomized controlled trial of motivational interviewing to prevent risk for an alcohol-exposed pregnancy in the Western Cape, South Africa. Addiction 2013; 108: 725-732.

25. Abrahams N, Jewkes R and Mvo Z. Health care-seeking practices of pregnant women and the role of the midwife in Cape Town, South Africa. J Midwifery Women's Health 2001; 46: 240-247. 